\title{
How to design a proper membrane for a membrane contactor-based air conditioning \\ system
}

\author{
Marta Bojarska \\ University of Duisburg-Essen \\ Universitätsstr. 7, 45141 \\ Essen, Germany \\ Email: marta.bojarska@uni-due.de
}

\author{
Stefano Lazzari \\ TICASS S.c.r.1. \\ via Bartolomeo Bosco, 57/4,16121 \\ Genoa, Italy \\ University of Genoa \\ Genoa, Italy \\ Email: stefano.lazzari@unige.it
}

\section{Gustavo Capanelli}

TICASS S.c.r.l.

via Bartolomeo Bosco, 57/4,16121

Genoa, Italy

Email: gustavo.capannelli@ticass.it

\author{
Clemens Alexowsky \\ University of Duisburg-Essen \\ Universitätsstr. 7, 45141 \\ Essen, Germany \\ Email: clemens.alexowsky@uni- \\ due.de
}

\section{Aldo Bottino}

TICASS S.c.r.l.

via Bartolomeo Bosco, 57/4,16121

Genoa, Italy

University of Genoa

Genoa, Italy

Email: bottino@chimica.unige.it

\author{
Mathias Ulbricht \\ University of Duisbur-Essen \\ Universitätsstr. 7, 45141 \\ Essen, Germany \\ Email: mathias.ulbricht@uni-due.de
}

\author{
Soccorso Gaeta \\ GVS Filter Technology \\ Via Roma 50, 40069 \\ Zola Predosa (Bologna), ITALY \\ Email: sng@gvs.com
}

\author{
Claudia Cattaneo \\ TICASS S.c.r.l \\ via Bartolomeo Bosco, 57/4,16121 \\ Genoa, Italy \\ Email: c.cattaneo@ticass.it
}

\begin{abstract}
This paper is dedicated to the development of proper membrane for membrane contactor-based air conditioning system. Based on mass transfer analysis and knowledge of membrane formation, guidelines for most appropriate membrane were made.
\end{abstract}

Keywords—gas liquid membrane contactor; air conditioning systems; air dehumidifaction; membrane formation.

Development of an energy friendly climate control system plays an important role in extending the range capability of electric vehicles in all weather conditions. During the XERIC (EU, HORIZON 2020) project, the usage of a novel Three Fluid Combined Membrane Contactor (3F-CMC) as an air conditioning system is explored. It is capable of reducing at least $50 \%$ of the energy used for passengers comfort, in comparison with existing systems. The 3F-CMC combines a liquid desiccant cycle with a traditional vapor compression cycle; moreover the full scale unit will be characterized by the following characteristics: external air flow rate $\left(\mathrm{T}=30^{\circ} \mathrm{C} ; \mathrm{RH}=60 \%\right.$ ) equal to $100 \mathrm{~m}^{3} / \mathrm{h}$, water vapor extraction from the external air of more than $0.3 \mathrm{~kg} / \mathrm{h}$, and a size of the unit equal to $310 \times 285 \times 185 \mathrm{~mm}$. The efficiency of such a system is mainly dependent on the module design and operation parameters, but performance of the membrane is also a crucial factor. In this work we will concentrate on the selection of the proper membrane for the membrane contactorbased air conditioning systems.

Membranes used in the 3F-CMC (or in other gasliquid membrane contactors with an aqueous liquid phase) should be characterized by performance values such as high water vapor transport rate, WVTR $\left(\mathrm{kg} / \mathrm{h} / \mathrm{m}^{2}\right)$, or low total resistance to the evaporative heat transfer, RET $\left(\mathrm{m}^{2} \mathrm{~Pa} \mathrm{~W} \mathrm{~W}^{-1}\right)$, as well as a high value of liquid entry pressure, LEP (bar). Those parameters describe possible operation pressure of the unit and expected performance in the external air dehumidification. Unfortunately, membrane manufacturers rarely use those parameters in membrane data sheets. In a typical commercial membrane data sheet, one can find information 
on membrane wettability, pore diameters, bubble point pressure, porosity, thickness etc. Also, the set of available data for a specific membrane differs depending on the intended membrane application. In this work, we try to link typical membrane characterization parameters with WVTR and LEP that are used in the performance description of the membrane contactor-based air conditioning system.

Based on the theoretical analysis of mass transfer in a flat sheet membrane, we were able to obtain the two following relationships:

WVTR $\sim \frac{d_{p} \varepsilon}{\tau \delta}$

$L E P \sim \frac{\cos \theta}{d_{p}}$

Where:

WVTR - water vapor transport rate $\left(\mathrm{kg} / \mathrm{h} / \mathrm{m}^{2}\right)$;

$\mathrm{dp}$ - mean pore size $(\mu \mathrm{m})$;

$\varepsilon-$ membrane porosity $(\%)$; $\tau-$ membrane tortuosity (-);

$\delta$ - membrane thickness $(\mu \mathrm{m})$;

LEP - liquid entry pressure (bar);

$\theta$ - contact angle $\left(^{\circ}\right)$.

Through comparison of the theoretical analysis with experimental data for commercial and self-made membranes, we will obtain the link between classical membrane characteristics and performance parameters relevant for the $3 \mathrm{~F}-\mathrm{CMC}$ unit. Based on the correlations, we will be able to design and prepare the membrane with properties that ensure a high performance of the $3 \mathrm{~F}-\mathrm{CMC}$ unit in the climate control system of (electric) vehicles.

\section{Acknowledgment}

This work was supported by the EU Horizon 2020 project "XERIC - Innovative Climate-Control System to Extend Range of Electric Vehicles and Improve Comfort".

DOI :10.1109/EVER.2017.7935952

Copyright @ 2017, IEEE 